\title{
The Design and Realization of Distance Education System Based on the Power Line Carrier
}

\author{
Chao Lv ${ }^{*}$
}

Computer Information Center, Beijing Institute of Fashion Technology, Beijing 100029, China

\begin{abstract}
Characterized by extensive coverage, easy connection and huge application potential, power line carrier communication has gained increasing attention and become a research hotspot in recent years. This paper started by an introduction of the power line carrier communication technology, and then compared it with other networking methods, finally practical power line networking project was proposed. Based on the advantages of power line communication technology, the hardware system was formatted applying four-tier B/S architecture. The computer distance education system was established using ASP technology in the premise of effective protection of network security. Study shows that the power line communication network is a reliable network education resource, of great significance to promote distance education.
\end{abstract}

Keywords: Power Line Carrier, Communication Technology, Distance Education, Design.

\section{INTRODUCTION OF POWER LINE COMMUNI- CATION TECHNOLOGY}

Power Line Communication (PLC) technology is a means of communication to transmit data and voice signals using low-voltage power line, referred to as PLC technology. In PLC system, the high frequency signal carrying information is loaded onto and transmitted by the power line, and separated through a dedicated power line modem, finally transmitted to the terminal device. PLC system mainly applies for data transmission within the scope of a transformer, extracting the electrical signal from the power line with the power line modem [1]. With the growing demand for highspeed Internet broadband communication, PLC technology will become one of the most competitive and potential broadband networking ways.

Power line Internet users need a special modem (PLC Modem) when the Internet connects to the distribution room of user-residential building via fiber-optic cables. The top of this modem data cable is a conventional power plug. What the user has to do to achieve Internet is: connect computer's network card and the modem, plug modem into the power outlet, and then connect to the PLC master device of building distribution transformers by the power line, now the connection of "the last mile" is completed [2].

\section{ADVANTAGES OF POWER LINE COMMUNICA- TION TECHNOLOGY}

The main advantages of transmitting data by PLC are: extensive coverage, convenience, no wiring, permanent online, low construction cost, short installation period, and realizing the "four -in-one" of data, voice, video and electric power without re-building network.

*Address correspondence to this author at the Computer Information Center, Beijing Institute of Fashion Technology, Beijing 100029, China; Tel: 1369306766; E-mail: lcrabbit@163.com

\subsection{Low Cost}

PLC is a "No New Wires" technology which takes full advantage of existing low-voltage distribution network infrastructure without any wiring, thus greatly reducing the line investment, saving cost, and avoiding some problems obtained from laying network line such as the destruction of the academic buildings and public facilities due to trenching and through-the-wall holes, as well as saving manpower [3]. PLC technology can provide high-speed Internet services to schools with much lower cost, which adds a new option for the "School-to-School" project, and helps speed up the IT application in education.

\subsection{Permanent Online}

PLC as a communication channel can flexibly achieve "plug and play" without cumbersome dialing process. It is always online as long as the power supply is being plugged. This characteristic of power line allows for building fireproofing and anti-theft security monitoring system at school and improving IT application.

\subsection{High Data Transfer Rate}

As for the technical properties, ADSL downlink speed is $8 \mathrm{MB} / \mathrm{s}$, HFC (Hybrid fiber coaxial cable) is $10 \mathrm{MB} / \mathrm{s}$, and the PLC transmission rate can reach $10 \mathrm{M}$ to $45 \mathrm{Mbps}$ (even $200 \mathrm{Mbps}$ in the future), totally a rival to ADSL and HFC. Compared with the telephone dialing and Integrated Services Digital Network (ISDN), PLC speed is even higher [4, 5]. The original power lines only transmit electric power rather than information, so the PLC can enjoy the entire signal bandwidth. While ADSL and HFC both need to take into account the telephone, television signals, which will be affected in the case of network congestion.

\subsection{Extensive Coverage}

At present, China has the world's second longest power transmission lines. Power lines of $500 \mathrm{KV}$ and $330 \mathrm{KV}$ have 
Table 1. Comparison of Power Line Networking and Other Networking Methods

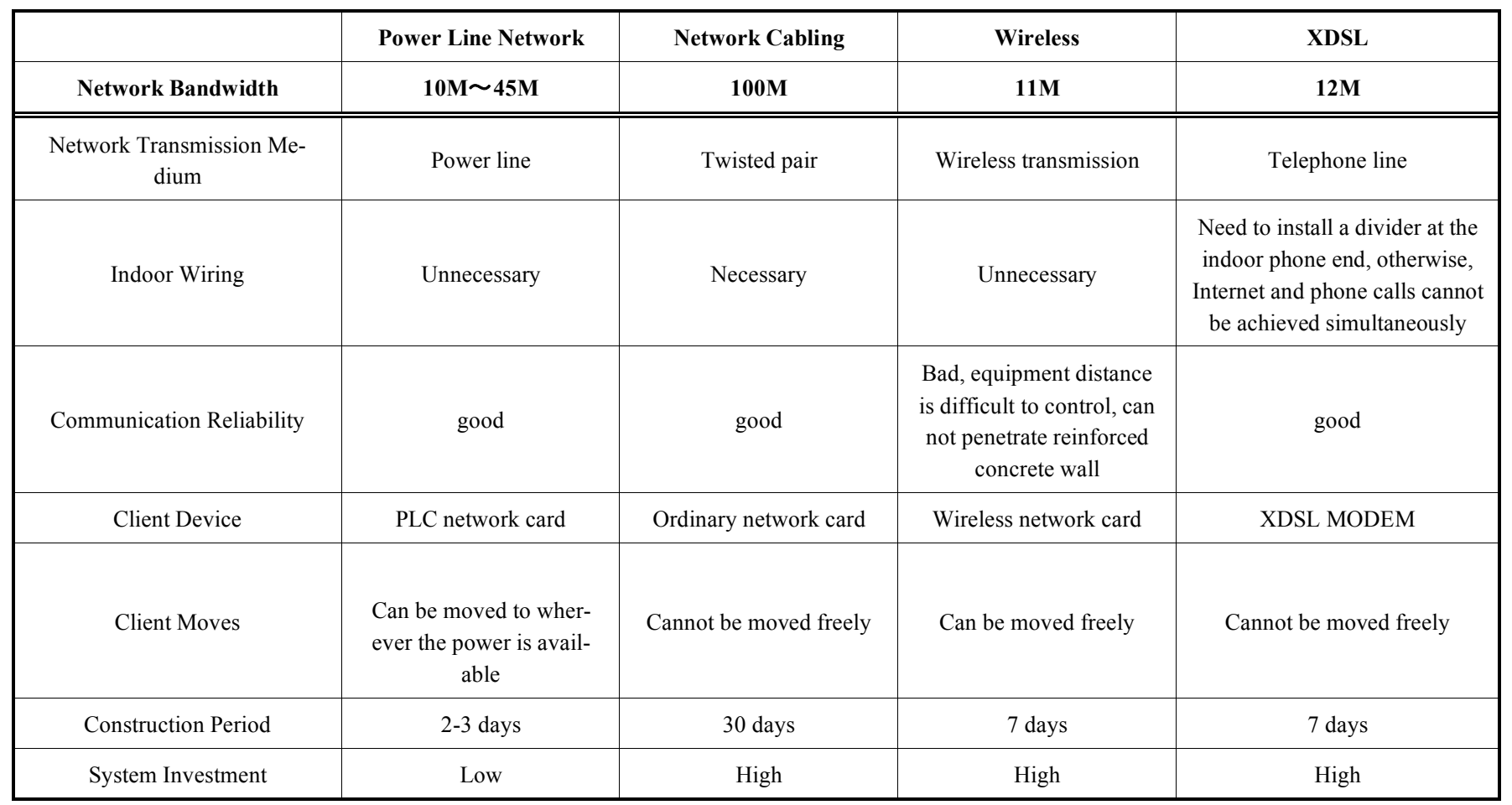

reached to $25094.16 \mathrm{~km}$, Power lines of $220 \mathrm{KV}$ have reached to $107348.06 \mathrm{~km}$, and total to $310000 \mathrm{~km}$ plus $110 \mathrm{KV}$ lines, nearly eight laps around the earth [6]. For each school, the electricity network is ready, and it does not need to re-build, where every classroom and every office have wire sockets. Even if in remote, outlying areas, accomplished first are still power lines. All of these make civilian power lines the world's most popular, most extensive coverage network, which is an advantage that telephone and cable TV cannot match.

\section{DESIGN PRINCIPLES OF THE SYSTEM}

\subsection{Progressiveness Principle}

The progressiveness principle can make distance education system relatively keep pace with era, in order to prolong the life cycle of the entire system as much as possible.

\subsection{Expansibility Principle}

Taking into account the medium-term and long-term expansion of the system, the system should adapt to the future needs of remote teaching and learning in the network architecture, network applications, network management, system performance, and other aspects.

\subsection{High Reliability Principle}

In the design, the distance education system based on PLC technology, the reliability points such as humancomputer security, long-term stable operation should be given top priority, to ensure the personal safety and system security during the use of the system. Upon the above reliability requirements being satisfied, the program should be optimized to reduce equipment investment as far as possible [7].

\subsection{Economically Practical Principle}

The design of the system should focus on economics and practicality, reduce overall cost, and seek the perfect union of the progressiveness and economics, making the distance education system more cost-effective.

\section{POWER LINE NETWORKING PROJECT}

\subsection{Comparison of Power Line Networking and Other Networking Methods (Table 1)}

\subsection{Feasibility Analysis}

Power line networking technology is primarily inserting a communication device (power line communication access device) into the output of the power line of the transformer on the user's end. The device can be connected to the mains via the twisted pair, wireless, XDSL, cable and optical fiber, to provide users with data, voice and multimedia services. Within the communication equipment, high-frequency network signal and low-frequency electrical signal are coupled to the power line of the user, and thus the communication network, the power distribution and the Customer Premise Network are connected. The electrical power wiring can be used to realize a home LAN: by connecting the computer, printer, telephone and fax machine and other equipment with ordinary power outlets.

The great advantage of power line network is low cost. Power line networking re-uses existing wiring directly and does not need extra wiring such as telephone lines or fiber optic cables, thus greatly reducing the investment of network construction. The equipment installation is simple, convenient, time-saving and flexible, especially suitable for network modification or extension. 


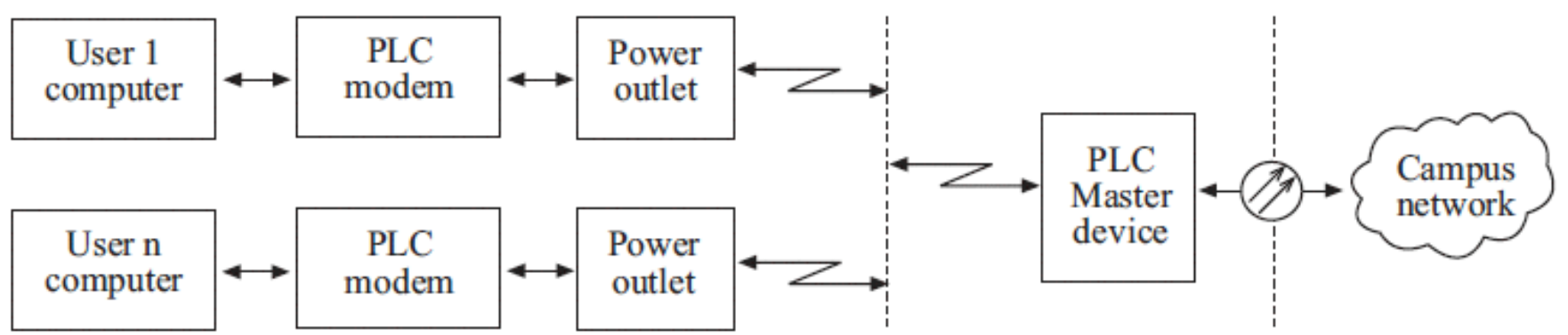

Fig. (1). Typical PLC system networking.

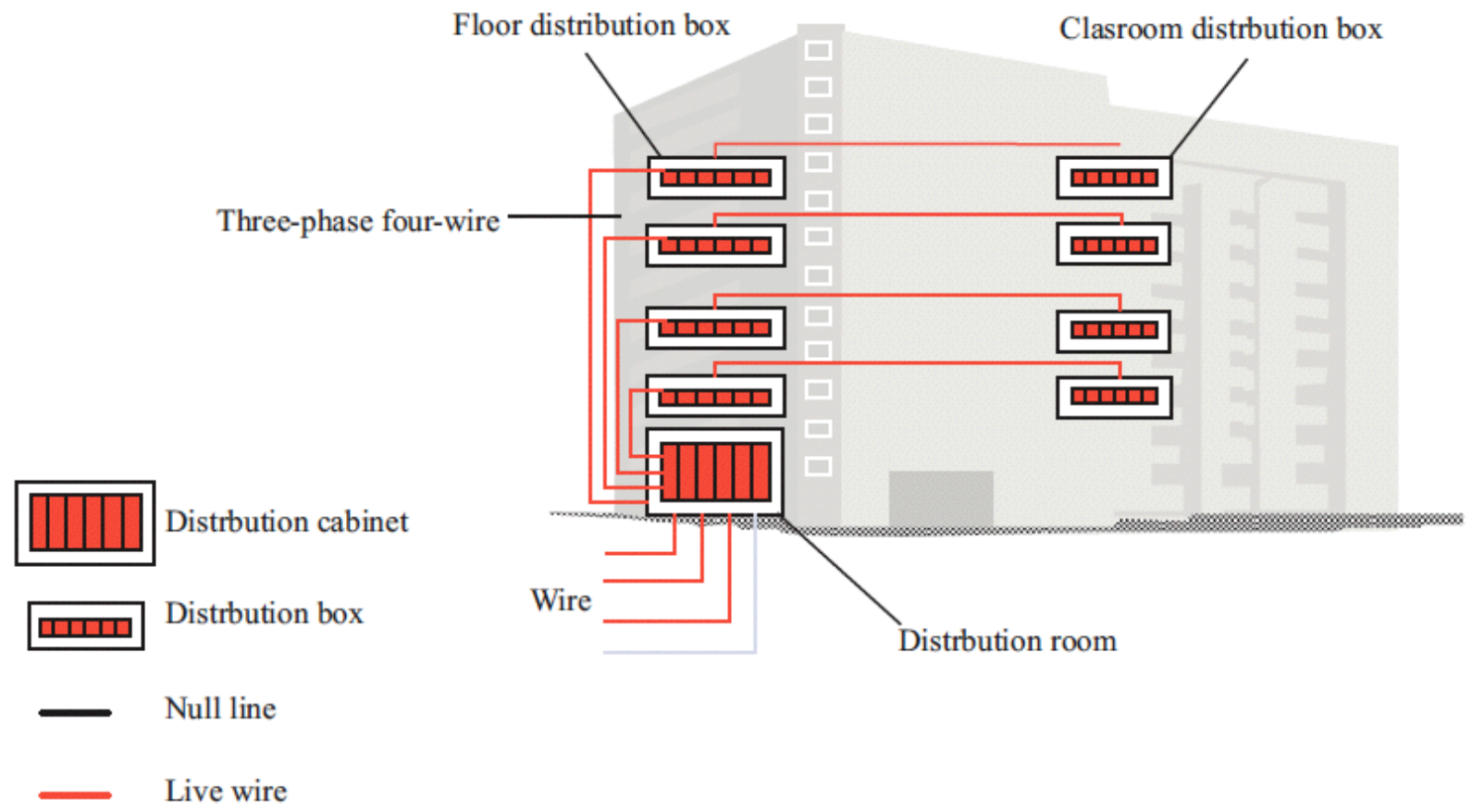

Fig. (2). Teaching building power line system distribution.

Using power line communication, users do not have to worry about the security of the electricity owing to the enough technical assurance, and the product will not cause any harm to the human body. In addition, the network security is guaranteed because of the encryption.

Internet connection via PLC allows flexible extension of the user ports, especially, this program does not need to consider the increasing or decreasing impact of the user on interface of the device, and the computer can be moved to any location without breaking the Internet.

PLC technology enables a higher communication speed. The $14 \mathrm{Mb} / \mathrm{s}$ chip of Intellon Corporation has reached the practical level, the European standard chip has reached $45 \mathrm{Mb} / \mathrm{s}$, and the speed can even reach $200 \mathrm{Mb} / \mathrm{s}$ through equipment upgrade in the future.

What the user has to do to achieve Internet is: connect computer's network card and the modem, plug modem into the power outlet, and then connect to the PLC master device of building distribution transformers by the power line, now the networking is completed.

Through the above analysis, we believe that the power line networking is feasible. A typical PLC system networking is shown in Fig. (1).

\subsection{Power Line Networking Project}

Let's assume that there is a four-layer teaching building with ten classrooms on each floor and the users who are on line at the same time are not so many. The users need to be given the functions like browsing the internet, downloading the resources and so on.

\subsubsection{Teaching Building Power Line System Distribution} (Fig. 2)

\subsubsection{Power Line Networking Topological Graph}

It adopts the PLC network bridges which will be installed on the first floor and the third floor. Each PLC network bridge can output four channels of high-frequency data signal. Through the ordinary civilian wires, the signal will be sent to the other two layers. The whole building will be covered just by two sets of PLC network bridges. One ethernet cable will be dragged from each network bridge to the network center in the teaching building and be connected with the switchboard to connect the whole power line network with the outer internet.

The structure of the whole system is simple and it is convenient to do installation. During the construction time, there is no need to run through the walls or dig the burrows to lay 


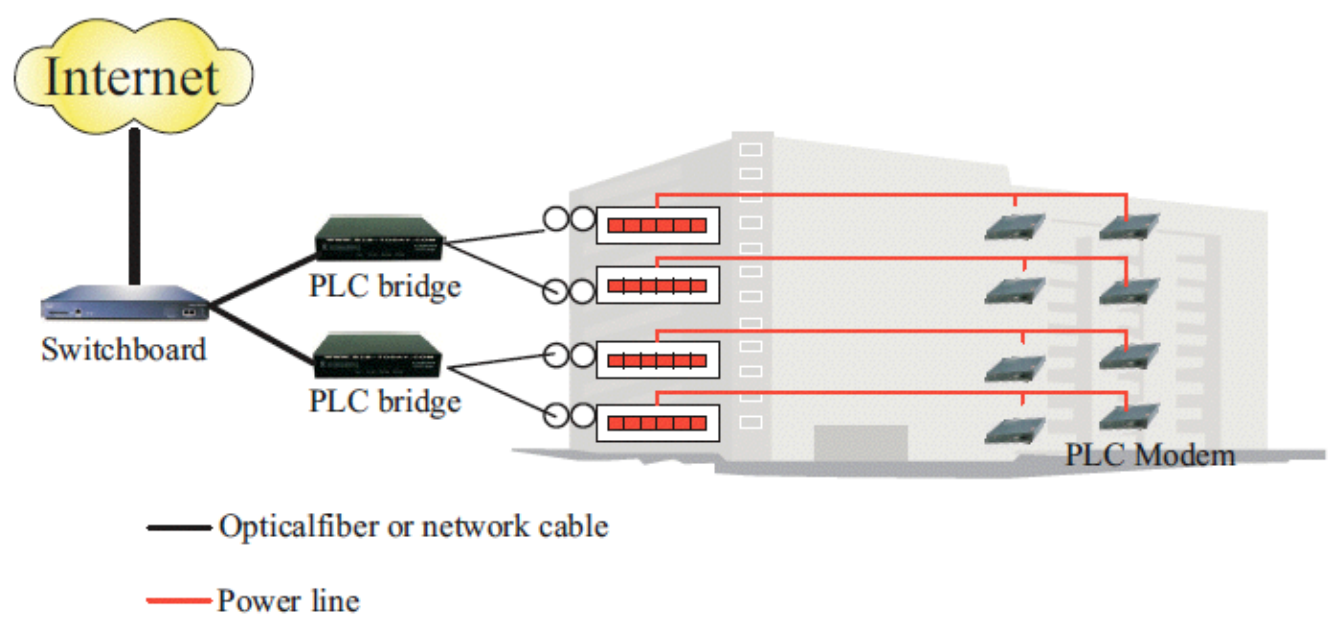

Fig. (3). Teaching building networking topological graph.

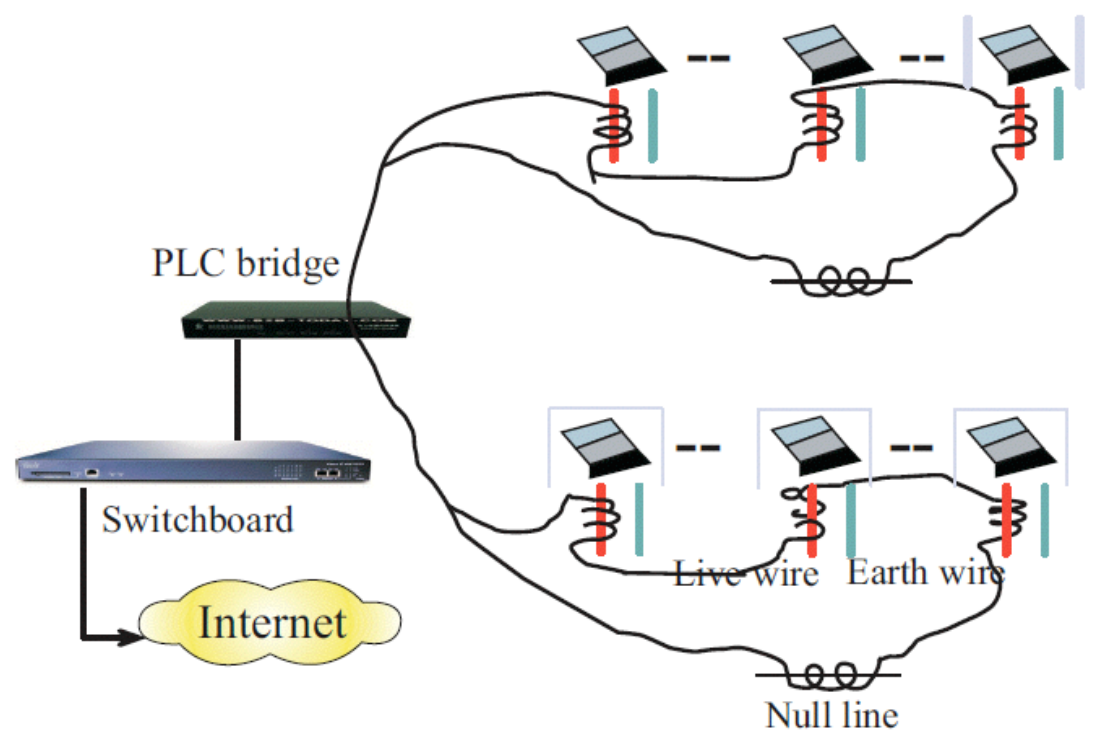

Fig. (4). The working drawing of power line network.

the lines. In the corridors and in the rooms on the each layer, it is unnecessary to horizontally lay any lines. When the users in each classroom are going to use the internet, what they only have to do is just plugging in the power resource of the PLC modem to any of the power sockets, and plugging the other end into the computer's USB port. Thus, the network of the whole building is linked. The topological structure of the network in the teaching building is shown in Fig. (3).

\subsubsection{The Working Drawing of Power Line Network (Fig. 4)}

In the distribution box on each floor, it will use the power lines which can transmit the signals and the filters with the Qualcomm magnetic core to couple the data signal with the power supply line in each classroom. In the whole construction progress, there is no need to change or add any equipment on the distribution line.

\subsection{Contrastive Evaluation}

Power line networking: 11 thousand Yuan (take example of configuring 20 PLC modems, as it is convenient to move, so the fixed installation is not needed).
Comprehensive wiring and networking: 22 thousand Yuan (count in 40 information points, not considering the destruction to the internal decoration of the building during the construction time of comprehensive wiring).

In conclusion, the best advantage of using the power line networking is that the investment of the equipment is far lower than that of other networking methods, and there is no need to worry about the destructive construction work made by the other networking methods. Especially when reforming and expanding the original computer network, or when doing digitalized transformation to the other networks (such as two-way television education system, video monitoring system and so on), the advantage of power line networking technology is incomparable to the other networking technology.

\section{THE DESIGN OF DISTANCE EDUCATION SYS- TEM BASED ON THE POWER LINE COMMUNICA- TION TECHNOLOGY}

\subsection{System Architecture of Distance Education System}

This system uses B/S (Browser / Server) architecture. In this architecture, the user interface is achieved through the 


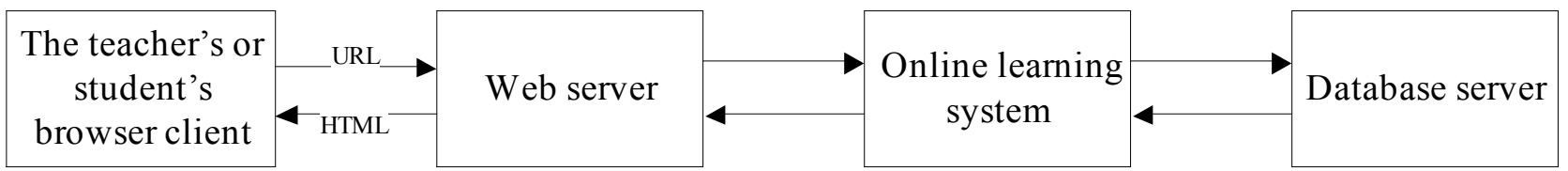

Fig. (5). System architecture of distance education system.

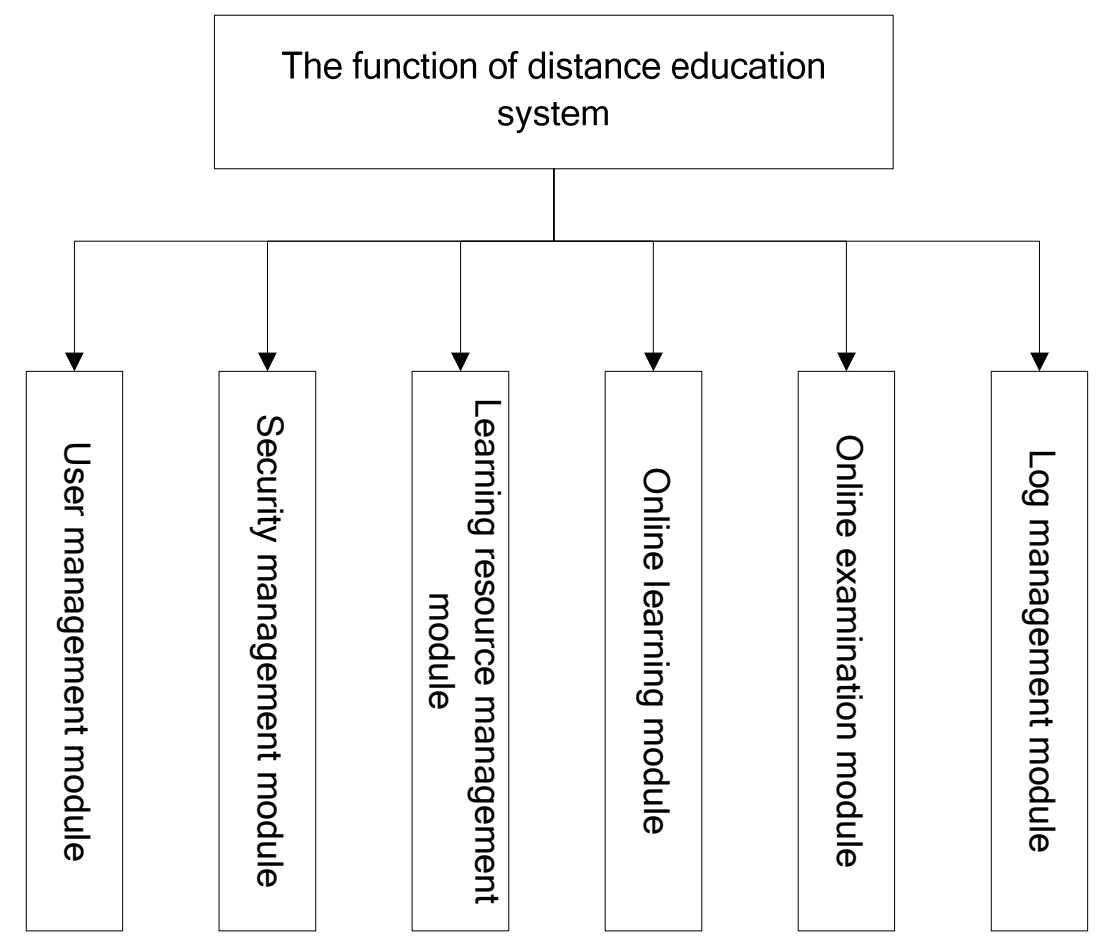

Fig. (6). The function of distance education system.

WWW browser, part of the business logic achieved at the front-end to, but the main business logic on the server end. $\mathrm{B} / \mathrm{S}$ architecture is divided into two kinds: the two-tier and three-tier. The two-tier architecture is composed of the browser and the Web server. In this architecture, the web page is static, incapable to change dynamically according to the user's action. The three-tier architecture is composed of the browser, web server and database server. Using the threetier architecture, data generation and data performance are integrated into a dynamic page, which makes the dynamic page become very large. In addition, the application performance and logic processing are mixed together, which brings many difficulties to the development and maintenance of Web application system [8]. To fix this problem, the application performance can be separated from logic processing to form a so-called four- tier architecture, as shown in Fig. (5). Web server (namely the layer where the dynamic page is) is mainly responsible for the application performance, while the application logic layer is mainly responsible for the logic processing of application.

\subsection{The Function of Distance Education System}

The distance education system based on power line communication technology is established using ASP technology, whose main function modules are shown in Fig. (6).

\subsubsection{User Management Module}

The main operations of the user management module include user creation, user login, user authorization and user change, user logout.

User creation: it needs the unit or the individual who uses distance education system to do application, and the manager will record the personal data and create a user's account and save it in the server. The user's account includes a unique username and a password that is related.

User authorization and user change: Through this operation, the manager will authorize the study material to the new users who are in need. In this way, the users who log in the study system can directly use their own authorized resources. User authorization (namely resource authorization) is not only a kind of user management operation, but also a kind of resource management operation. In the distance education system, adopting the policy of resource access control based on the role can simplify the authorization operation and be convenient for resource management.

User login: a user with the legal account can enter into the distance education system by inputting his own username and password on the login screen. 


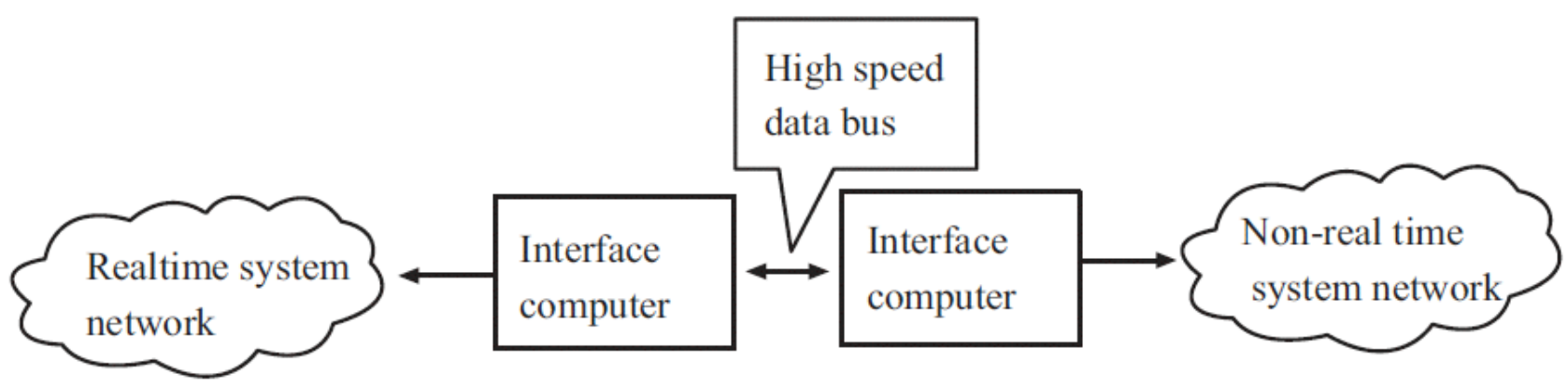

Fig. (7). The double host isolation firewall.

User logout: After user finishes the learning tasks, he does logout actions to delete the user account from the server. User logout means that the life cycle of the user is over.

\subsubsection{Security Management Module}

In the network environment, security management is very important. In the design of distance education system, security management function should be provided. The system uses the software function to set up different limits of authority, such as system administrator privileges, teacher privileges and student privileges, etc., and the new student can become an official student only after his registration was confirmed by the relevant departments, and then can get the relevant information. The double host isolation firewall adopted by the system is shown in Fig. (7). When it is working, configuring access rules that meet the security strategy on the firewall can check the data that go through the internal and external network and prevent the unauthorized access from going through the firewall. When sending data between the realtime system and the non-realtime system, it adopts routing mechanism and uses the high speed data bus among the interface computers to make connection and transmission and prevents the users in the company and a few external personnels who successfully pass through the firewall from using the non-realtime system to attack the realtime system.

\subsubsection{Learning Resource Management Module}

In the network environment, various kinds of resources are coexisting in the distance education system and are constantly changing. The system will provide the administrator or the authorized user a unified platform and fulfill the management of the resource information. The main administrative operations designed here include registering, modifying, deleting, inquiring and authorizing the resource objects, etc [9]. Among them, the operation of authorizing the resource objects exclusively belongs to the administrator; the operation of registering, modifying and deleting the resource objects belongs to the administrator and the teacher; the operation of inquiring the resource objects belongs to all the users.

\subsubsection{Online Learning Module}

Online learning module is used for students to choose learning content, download the schoolwork, ask questions to the teacher, discuss with the teacher and other students in real time, read bulletin, complete and submit schoolwork online, query schoolwork correcting status and test scores, and so on.

\subsubsection{Online Examination Module}

Online examination module is used for the assessment, results query, selecting the databank of examination questions to generate papers automatically and preforming papers for assessment.

\subsubsection{Log Management Module}

In order to guarantee the normal operation of the system and accurately solve various kinds of system problems that may meet, having a good log management is very important. In the progress of using the system, error message, warning message and other messages may be caused because of some reasons. These messages are very important, so they shall be written in a file, which is called the function of log management [10]. In the education system, the log is indicated with a text file. Each line in the file is a message. Each message mainly consists of the time when the message generates, the server name that generates the message and the specific content of the message. Through managing and checking the log files, we can better maintain the system and guarantee that various kinds of application are running normally.

\subsection{The Realization of Software System}

The development of B/S structural system is mainly applied to the ASP technology. ASP comprehensively uses the traditional HTML file, ActiveX script language and the ActiveX component technology [11]. It executes the script and applies the ActiveX service component at the server side. When the user is working, the screen browsed through the browser is the result returned by the ASP interpreter after its execution, which is applicable to any kinds of browsers. ASP interpreter (ASP.DLL) is integrated in the ISS server in the form of ISAPI application program.

The database access component, ADO (ActiveX Data Object), the built-in component in the ASP is applied to access the database. ADO includes multiple objects like Connection, RecordSet and so on. Generally, it is through the Connection to make connection with the database, and sometimes, it can directly use the RecordSet to access the database according to the need. Before using the ASP component, it shall create this component at first, and then, it can invoke the ASP component and access its attributes.

\section{CONCLUSIONS}

This paper analyzes the advantages of power line communication technology, and then compares it with other networking methods, finally practical power line networking 
project is proposed. It is proposed that hardware system can be developed using four-tier $\mathrm{B} / \mathrm{S}$ architecture, and PLCbased distance education system can be established applying ASP technology, in consistence with progressiveness, expansibility, reliability and practicality principles. The distance education system can help achieve learning resources management, user management, online examinations and other functions. In the vast non-central areas and the rural areas, the PLC has certain advantages, which is of great significance in promoting distance education.

\section{ACKNOWLEDGEMENTS}

This work was financially supported by Beijing municipal universities academic innovation team project (RCQJ02120207).

\section{REFERENCES}

[1] Q.S. Qi, "Power Line Communication Technology and Application", China: China Electric Power Press, 2005.

[2] C. Lv and Y. Peng, "The Application of Power Line Communication Technology in Campus Network Construction", The 9th International Forum of Educational Technology, pp. 275-279, 2010.
[3] L.H. Ma, "Analysis and Application of Power Line Communication (PLC)", Jisuanji Yu Xiandaihua, vol. 4, pp. 42-44, 2003.

[4] Z.P. Zhang and Y.Z. Zhao, "Comparison of communication method based on low power line carrier", Electronic Design Engineering, vol. 18, no. 2, pp. 26-28, 2010.

[5] L.X. Wu, W. Zhang and Z.S. Hou, "Power line access technique and AN. s development", Telecommunications for Electric Power System, vol. 11, p. 1-4, 2001.

[6] H.T. Chen, "Application in broadband access of PLC", Journal of Beijing Technology and Business University, vol. 23, no. 2, pp. 52$55,2005$.

[7] C. Lv, "Design and Realization of Power Line Modem with Function of Protocol Conversion", M.S. thesis, Department of Educational Technology, Capital Normal University, Beijing, China, 2005.

[8] S.H. Zhang and Y.Z. Zhu, "The 4-tier Browser / Server Architecture and Its Scheme", Application Research of Computers, vol. 9, pp. 21-22, 2002.

[9] Y. Wang, S.H. Shi and S.J. Gong, "Web-based Intelligent Distance Education System", E-Education Research, vol. 8, pp. 33-36, 2005.

[10] F.Y. Liu, "The Portal Designing of Distant Learning System Base on Grid", M.S. thesis, IT Engineering College, Central South University, Hunan, China, 2006.

[11] G.Z. Zhang, and K. Yang, "Application of ASP. NET Technique in Rural Distance Education System”, Journal of Anhui Agricultural Sciences, vol. 35, no. 9, pp. 2820-2821, 2007.

(C) Chao Lv; Licensee Bentham Open.

This is an open access article licensed under the terms of the Creative Commons Attribution Non-Commercial License (http://creativecommons.org/licenses/by-nc/3.0/) which permits unrestricted, non-commercial use, distribution and reproduction in any medium, provided the work is properly cited. 